\title{
Bone Turnover Markers After Six Nights of Insufficient Sleep and Subsequent Recovery Sleep in Healthy Men
}

\author{
Christine M. Swanson ${ }^{1}(1) \cdot$ Prajakta Shanbhag $^{2} \cdot$ Emma J. Tussey $^{2} \cdot$ Corey A. Rynders ${ }^{2} \cdot$ Kenneth P. Wright Jr ${ }^{1,3}$. \\ Wendy M. Kohrt ${ }^{2}$
}

Received: 1 November 2021 / Accepted: 22 January 2022 / Published online: 8 February 2022

(c) The Author(s), under exclusive licence to Springer Science+Business Media, LLC, part of Springer Nature 2022

\begin{abstract}
Purpose The goal of this study was to determine the bone turnover marker (BTM) response to insufficient and subsequent recovery sleep, independent of changes in posture, body weight, and physical activity.

Methods Healthy men $(N=12)$ who habitually slept $7-9 \mathrm{~h} /$ night were admitted to an inpatient sleep laboratory for a baseline $8 \mathrm{~h} /$ night sleep opportunity followed by six nights of insufficient sleep $(5 \mathrm{~h} / \mathrm{night})$. Diet, physical activity, and posture were controlled. Serum markers of bone formation (osteocalcin, PINP) and resorption ( $\beta$-CTX) were obtained over $24 \mathrm{~h}$ at baseline and on the last night of sleep restriction, and on fasted samples obtained daily while inpatient and five times after discharge over 3 weeks. Maximum likelihood estimates in a repeated measures model were used to assess the effect of insufficient and subsequent recovery sleep on BTM levels.

Results There was no statistically or clinically significant change in PINP $(p=0.53)$, osteocalcin $(p=0.66)$, or $\beta$-CTX $(p=0.10)$ in response to six nights of insufficient sleep. There were no significant changes in BTMs from the inpatient stay through 3 weeks of recovery sleep (all $p \geq 0.63$ ). On average, body weight was stable during the inpatient stay $(\Delta$ weight $=-0.55 \pm 0.91 \mathrm{~kg}, p=0.06)$.

Conclusion No significant changes in serum BTMs were observed after six nights of insufficient or subsequent recovery sleep in young healthy men. Changes in weight and physical activity may be required to observe significant BTM change in response to sleep and circadian disruptions.
\end{abstract}

Clinical Trials Registration Registered at ClinicalTrials.gov (NCT03733483) on November 7, 2018.

Keywords Sleep restriction $\cdot$ Bone turnover markers $\cdot$ Insufficient sleep $\cdot$ Recovery sleep

\section{Abbreviations}

BTM Bone turnover marker

h Hour

PINP N-terminal propeptide of type I procollagen

$\beta$-CTX C-telopeptide of type 1 collagen

Christine M. Swanson

Christine.Swanson@CUAnschutz.edu

1 Division of Endocrinology, Metabolism and Diabetes, Department of Medicine, University of Colorado Anschutz Medical Campus, 12801 E. 17th Ave, Mail Stop 8106, Aurora, CO 80045, USA

2 Division of Geriatric Medicine, University of Colorado Anschutz Medical Campus, and Eastern Colorado VA Geriatric, Research, Education, and Clinical Center (GRECC), Aurora, CO, USA

3 Sleep and Chronobiology Laboratory, Department of Integrative Physiology, University of Colorado Boulder, Boulder, CO, USA

$\begin{array}{ll}\text { BMD } & \text { Bone mineral density } \\ \Delta & \text { Change }\end{array}$

\section{Introduction}

Insufficient sleep duration is a risk factor for many cardiometabolic diseases including diabetes mellitus type 2, obesity, coronary heart disease, and all-cause mortality [1-11]. Emerging literature, primarily from pre-clinical models, suggests insufficient sleep duration may also influence bone metabolism $[12,13]$. Two studies of chronic sleep restriction in rats demonstrated declines in markers of bone formation with either no change or an increase in markers of bone resorption [12, 13]. As might be expected from this detrimental uncoupling of bone turnover markers where resorption exceeds formation, these studies also demonstrated lower bone mineral density (BMD) and poorer 
bone microarchitecture in sleep-restricted rats compared to controls [12, 13]. In humans, cross-sectional studies have identified associations between short sleep duration and low BMD [14-17]. The Women's Health Initiative (WHI) identified increased fracture risk with sleep duration of $\leq$ $5 \mathrm{~h} /$ night [18]. Although associations have also been identified for long sleep duration with both low BMD and fracture risk [17-19], short sleep duration is more commonly experienced. In fact, the CDC highlighted insufficient sleep as a public health epidemic in 2014 [20], with over onethird of U.S. adults reporting less than $7 \mathrm{~h}(\mathrm{~h})$ per night [21]. Prior human intervention studies identified a decline in serum markers of bone formation in response to severe sleep restriction ( $2 \mathrm{~h} / \mathrm{night})$ [22] and, in a separate study, 3 weeks of cumulative sleep restriction $(\sim 5.5 \mathrm{~h}$ per $24 \mathrm{~h})$ with concurrent circadian disruption [23, 24]. However, the effects of modest, commonly experienced insufficient sleep duration (e.g., $5 \mathrm{~h}$ /night), mechanisms by which insufficient sleep affects bone metabolism, and the ability to recover from these exposures with sleep extension had not been studied.

The goal of the present study was to determine the bone turnover marker (BTM) response to sleep restriction $(5 \mathrm{~h} /$ night for six nights) and subsequent recovery sleep, independent of changes in body weight, posture, or physical activity. Based on our prior work [23, 25] and animal data $[12,13]$, we hypothesized that six nights of insufficient sleep duration would cause declines in serum markers of bone formation (osteocalcin; N-terminal propeptide of type I procollagen, PINP) but not a marker of bone resorption (C-telopeptide of type I collagen, $\beta$-CTX) and that bone formation marker levels would return to baseline within 3 weeks of resuming a normal sleep/wake schedule.

\section{Methods}

\section{Study Design and Participant Selection}

Participants were recruited from the Denver, Colorado area between January 2019 and January 2020 using flyers, electronic advertising, and social media marketing. The protocol was performed in twelve healthy men who habitually slept 7-9 h/night and consisted of three consecutive phases: outpatient habitual controlled sleep duration during a run-in week, inpatient baseline night followed by sleep restriction, and observational recovery phase. The protocol was performed in men because when the study was designed, data demonstrating changes in BTMs in response to sleep interventions were available only in men [23, 26] and male animals [12]. All men were healthy as determined by questionnaires, labs (including a urine drug screen), BMD screening by dual-energy $\mathrm{x}$-ray absorptiometry (DXA), history, and physical exam prior to study entry. All men were screened using the Berlin questionnaire and a WatchPat [27] home sleep apnea testing device to exclude for moderate-to-severe sleep apnea. Participants were free of self-reported clinically significant sleep disorders or medical conditions and took no prescription medications.

To be eligible for the study, participants could not routinely go to bed after midnight, have a history of night shift work in the past year, have lived outside the Denver area (i.e., not lived at altitude) in the past 3 months, or have traveled $>1$ time zone away in the past 4 weeks.

During the outpatient habitual sleep duration run-in phase, all participants maintained a consistent 8-h sleep opportunity at their habitual bed/wake time, verified by wrist actigraphy, sleep diary, and time-stamped texts (Fig. 1). Immediately following the outpatient week, participants were admitted to the University of Colorado Clinical and Translational Research Center (CTRC) inpatient sleep suite for an 8-night inpatient stay. The first night was a baseline night with a habitual 8-h sleep opportunity, during which blood was obtained hourly for $24 \mathrm{~h}$. On nights $2-7$, participants were sleep restricted to a 5-h sleep opportunity per night. Sleep was shortened by delaying bedtime and advancing wake time by $1.5 \mathrm{~h}$ each. On night 7 , the hourly blood draws were repeated for $24 \mathrm{~h}$, at the same time points as on night 1. All 24-h blood profiles were aligned across and within participants to the midpoint of sleep. Fasted blood was also collected on days 2-6 and on day 9 at habitual wake time. On nights 2-7 when sleep restriction was imposed, participants remained semi-recumbent (head of bed raised $35^{\circ}$ ) for the $1.5 \mathrm{~h}$ before bedtime and $1.5 \mathrm{~h}$ after wake time to control for postural changes between conditions that may contribute to BTM changes, while helping to maintain wakefulness. For safety, participants were provided with a $\geq 10-\mathrm{h}$ sleep opportunity after the last 24-h blood draw was completed on night 8 prior to discharge on day 9. Participants had 24/7 supervision during both $24-\mathrm{h}$ blood draws and constant supervision while awake throughout the inpatient phase to ensure adherence to the protocol. There was one protocol deviation in which one participant slept $\sim 1 \mathrm{~h}$ longer than prescribed on night 5 of sleep restriction.

After discharge from the inpatient unit, participants could opt in for an observational recovery phase to investigate the potential time course of reversibility of sleep restriction-induced changes in BTMs. During this 3-week observation phase, participants wore the physical activity tracking devices and maintained a sleep log but were not given instructions on sleep duration or timing. Morning fasted blood was to be obtained on days $1,2,6,13$, and 20 after discharge, but approximately $33 \%$ of these draws occurred on different days due to scheduling issues. One participant declined to participate in this portion of the 


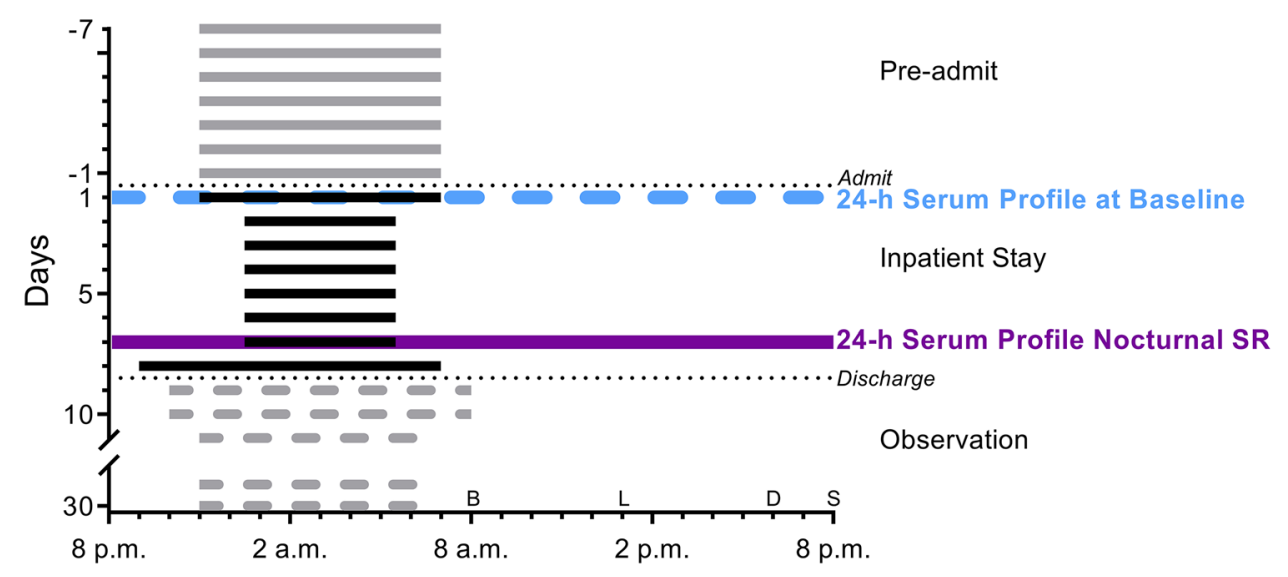

Approximate Relative Clock Time

Fig. 1 Study protocol. $X$-axis represents approximate relative clock time based on average bedtime and waketime of participants selfselected 8-h sleep opportunity. Horizontal gray bars represent outpatient 8-h sleep opportunities prior to admission. Horizontal black bars represent inpatient sleep opportunities. Horizontal dotted gray bars represent theorized sleep schedules during 3-week outpatient observational segment of the protocol with catch-up sleep in the days

protocol. Three of the men missed one draw each due to scheduling issues and the COVID-19 pandemic.

All participants provided informed consent. The protocol was approved by the Colorado Multiple Institutional Review Board (COMIRB) and registered at ClinicalTrials.gov (NCT03733483).

\section{Participant Characteristics}

Race/ethnicity was self-reported using the standard NIH categories. Body mass index (BMI) was calculated using height and fasted weight obtained at the screening visit using a stadiometer and digital scale. Serum calcium and creatinine were measured at the University of Colorado Hospital clinical and reference laboratory using indirect ion selective electrode and colorimetry, respectively, at the screening visit to evaluate for underlying abnormalities that could affect study results (e.g., chronic kidney disease). To qualify for the study, participants' calcium and creatinine at the screening visit had to be within the lab's normal range $(8.6-10.3 \mathrm{mg} / \mathrm{dL}$ and $0.70-1.30 \mathrm{mg} /$ $\mathrm{dL}$, respectively) with an estimated glomerular filtration rate (eGFR) $\geq 60 \mathrm{~mL} / \mathrm{min} / 1.73 \mathrm{~m}^{2}$ using the IDMS-traceable CKD-EPI equation for ages 18-97 years old used by the University of Colorado clinical laboratory. To be enrolled in the study, participants had to have a negative urine drug screen at both the screening visit and inpatient admission. after discharge and then resumption of habitual sleep/wake schedule. Dotted black lines represent when participants were admitted to and discharged from the inpatient stay. Dotted light blue horizontal line indicates baseline 24-h serum collection. Solid purple horizontal line represents 24-h serum collection during nocturnal sleep restriction (SR). Letters along the $x$-axis represent inpatient meal times for breakfast (B), lunch (L), dinner (D), and snack (S)

\section{Wrist Actigraphy and Outpatient Sleep Data Collection}

Bedtime, wake time, and sleep duration were tracked in multiple ways during outpatient phases. All participants wore a wrist actigraphy device (ActiWatch2 [28]) on their non-dominant wrist before, during, and after admission to track sleep/wake and physical activity levels and patterns. Participants were instructed to push the ActiWatch2 event marker at bed and wake times. Participants also completed a sleep diary during outpatient phases. During the outpatient run-in phase, bed and wake times were also verified with time-stamped, secure texts. For time-stamped texts, participants texted "bedtime" or "wake time" to a secure Google voice phone number when they went to sleep and woke up, respectively. These texts were stamped with the time they were sent and were used to track each participant's sleep timing and duration and to ensure adherence to the outpatient protocol. Average bed and wake times reported via text from the run-in week were used to determine habitual bed and wake times for the inpatient week.

\section{Polysomnography}

During the two 24-h blood collection intervals, sleep was verified by the Siesta 802 polysomnography device (Compumedics USA Inc). Polysomnography electroencephalogram (EEG), bilateral submental electromyographic (EMG), and bilateral electrooculographic (EOG) electrodes were applied 
by study staff using tensive adhesive gel. The following EEG channels were obtained: F3/A2, C3/A2, C4/A1, and O1/A2. $\mathrm{CZ}$ and $\mathrm{FZ}$ were used as reference and ground electrodes, respectively. Polysomnography sleep stages were scored by one trained scorer using AASM guidelines [29].

\section{Bone Turnover Markers (BTMs)}

Blood was processed immediately after collection and stored at $-80{ }^{\circ} \mathrm{C}$ until thawed for assays. All samples from an individual were run in the same assay to minimize variability. C-telopeptide of type I collagen ( $\beta$-CTX) was used as a marker of bone resorption. Osteocalcin and intact $\mathrm{N}$-terminal propeptide of type I procollagen (PINP) were used as markers of bone formation. Serum samples were assayed in singleton at the University of Colorado CTRC Core laboratory, which provided inter- and intra-assay coefficients of variation (CV). $\beta$-CTX, PINP, and osteocalcin were measured by chemiluminescence using the automated IDS iSYS instrument (Immunodiagnostics Systems, United Kingdom). For $\beta$-CTX, interassay CV was $8.6 \%$ at $196 \mathrm{ng} / \mathrm{L}$ and $6.4 \%$ at $406 \mathrm{ng} / \mathrm{L}$ and $5.8 \%$ at $2080 \mathrm{ng} / \mathrm{L} . \beta-\mathrm{CTX}$ intraassay CV was $7.7 \%$ at $201 \mathrm{ng} / \mathrm{L}, 4.6 \%$ at $446 \mathrm{ng} / \mathrm{L}$, and $3.4 \%$ at $2050 \mathrm{ng} / \mathrm{L}$. PINP interassay $\mathrm{CV}$ was $5.6 \%$ at $20.36 \mathrm{g \mu} / \mathrm{L}$, $3.5 \%$ at $51.23 \mathrm{g \mu} / \mathrm{L}$, and $1.7 \%$ at $175.21 \mathrm{g \mu} / \mathrm{L}$. PINP intraassay $\mathrm{CV}$ was $4.5 \%$ at $2.77 \mathrm{~g} \mu / \mathrm{L}, 1.9 \%$ at $37.24 \mathrm{~g} \mu / \mathrm{L}$, and $2.0 \%$ at $175.84 \mathrm{~g} \mu / \mathrm{L}$. Osteocalcin inter-assay $\mathrm{CV}$ was $1.9 \%$ at $7.95 \mathrm{ng} / \mathrm{mL}, 3.9 \%$ at $61.73 \mathrm{ng} / \mathrm{mL}$, and $5.5 \%$ at $138.20 \mathrm{ng} /$ $\mathrm{mL}$. Osteocalcin intra-assay $\mathrm{CV}$ was $4.0 \%$ at $2.72 \mathrm{ng} / \mathrm{mL}$, $1.7 \%$ at $23.70 \mathrm{ng} / \mathrm{mL}$, and $2.4 \%$ at $137.65 \mathrm{ng} / \mathrm{mL}$.

\section{Heparin}

Low-dose heparin (4000 units/L normal saline) was used to facilitate overnight blood draws through a long intravenous (IV) line that passed through a port in the wall of the inpatient room while the participant was sleeping so as not to disturb sleep. A blood sparing sampling method with Vamp syringe tubing was used for blood draws performed with the long IV tubing (volume $5 \mathrm{cc}$ ) with multiple sampling ports. With this procedure, $10 \mathrm{cc}$ of "waste" were drawn through the long IV tubing into a syringe, the blood sample was subsequently drawn from another port located closer to the participant's IV site, and after the sample was obtained the $10 \mathrm{cc}$ of "waste" were returned to the participant. The long IV tubing was flushed for over $1 \mathrm{~min}(\sim 20 \mathrm{cc}$ of saline) to completely clear the line of any residual heparin before the sample was drawn and again after the sample was obtained, before the heparin was turned back on. Heparin and long IV tubing were only used during sleep opportunities. Therefore, heparin exposure was $3 \mathrm{~h}$ longer during the baseline 24-h collection compared to the sleep-restricted $24-\mathrm{h}$ collection.

\section{Sleep Questionnaires}

All participants completed the Pittsburgh Sleep Quality Index (PQSI) [30] during screening to evaluate for subjective sleep complaints. Participants were excluded based on a global PSQI score $>5$ indicating poor sleep quality. During the study, subjective sleepiness was assessed with the Karolinska Sleepiness Scale (KSS) three times a day (30 min after habitual wake time, $5.5 \mathrm{~h}$ after wake, and $30 \mathrm{~min}$ before habitual bedtime). The Epworth Sleepiness Scale [31, 32] was performed at screening (during which individuals with a score $>10$ were excluded), the first day of the outpatient phase and on days 1,2 , and 8 of the inpatient phase.

\section{Physical Activity}

Physical activity levels and patterns for a given individual were matched during their outpatient and inpatient weeks using wrist actigraphy (ActiWatch2) and a Fitbit Charge 3 device to monitor for differences in physical activity that could confound the results. In addition, physical activity was standardized between individuals by having each participant perform three, 20-min walking bouts daily during the outpatient run-in and inpatient weeks. To ensure protocol adherence during the outpatient run-in week, participants texted the same secure, time-stamped phone number that was used to track sleep times when they completed their daily walking bouts. Physical activity (in arbitrary activity counts) was tracked with the ActiWatch2 [28]. Actigraphy data were cleaned and analyzed according to the MESA scoring manual [33]. Participants also wore a Fitbit Charge 3 device to track step counts to facilitate physical activity matching between outpatient and inpatient weeks by allowing for remote monitoring and check-ins throughout the day. Fitbit data from the outpatient run-in phase were used to match participant step counts $( \pm 10 \%)$ for average weekday and weekend days. In addition, workouts participants completed during the outpatient run-in week were recorded and matched during the inpatient week using the research gym attached to the inpatient unit.

\section{Diet}

Meals were provided by the CTRC Nutrition Core for the last three days of the outpatient week leading up to admission and throughout the inpatient stay. Caloric needs were determined using the Mifflin-St. Jeor Equation for men based on a participant's age, height, weight, and an activity factor of 1.4-1.7 (varied depending on participant's selfreported activity and Fitbit-recorded activity during the outpatient run-in week) [34]. Participants received their calories in three meals (breakfast, lunch, dinner) and an after-dinner snack (Fig. 1). Participants received 7\% more 
calories on sleep-restricted days to compensate for increased energy expenditure [35, 36], which were distributed across all meals. Three men required additional food during sleep restriction due to hunger. Fasted morning weights were obtained daily at habitual wake time, after the fasted morning blood draw. Macronutrient content was 55\% carbohydrate, $30 \%$ fat, and $15 \%$ protein. Diet was controlled for calcium (1000 mg $\pm 100 \mathrm{mg}$ per day) and phosphorous content (1600 mg $\pm 100 \mathrm{mg}$ per day). Meal times were standardized to occur at $12 \mathrm{~h}$ (breakfast), $17 \mathrm{~h}$ (lunch), $22 \mathrm{~h}$ (dinner), and $24 \mathrm{~h}$ (snack) into the sampling profiles during the inpatient phase. Caffeine was not permitted during the outpatient habitual sleep duration phase or inpatient sleep restriction phase. Participants had to report habitual alcohol use during screening and only those with $\leq 14$ alcohol drinks per week and $\leq 5$ drinks in one sitting were eligible to participate. Alcohol was not explicitly prohibited during the outpatient habitual sleep duration phase. However, participants were instructed to only consume food and beverages (other than water) provided by the CTRC Nutrition Service three days prior to and throughout inpatient admission. No alcohol was provided with the CTRC meals.

\section{Light Exposure}

No light control was included in this protocol. Lights in the CTRC inpatient unit are 28 watts in the $3500-4100 \mathrm{k}$ spectrum. From the angle of gaze on the inpatient bed, the typical lux levels in the sleep suite on cloudy and sunny days were 198.7 lx and 1200 lx, respectively. A blackout shade was used during sleep periods and lights were controlled from outside the room. During lights-out sleep periods, typical lux was 0.6.

\section{Daylight Saving Time (DST)}

Only one participant was studied around a DST transition. In that case, DST ended on the third day of their outpatient habitual sleep duration run-in week. To keep light exposure and circadian alignment consistent throughout the duration of the study, bed and wake times after DST ended were moved $1 \mathrm{~h}$ earlier than habitual (e.g., $11 \mathrm{pm}$ habitual bedtime pre-DST end and $10 \mathrm{pm}$ bedtime post-DST end).

\section{Statistical Analysis}

Using a paired $t$ test and prior data [23], we had $>80 \%$ power to detect a change in PINP of $\geq 10 \%$ (assuming 11\% variability in the percent change, a correlation of 0.5 between measures on the same participant, and a 2-sided alpha $=0.05$ ). Similar to prior analyses [23, 24], maximum likelihood estimates in a repeated measures model was used to assess the effect of insufficient and subsequent recovery sleep on
BTM levels, accounting for potential within-participant correlation. This was done by evaluating the test statistic $\mathrm{F}$ for equality of means using the ratio of the variation due to insufficient and subsequent recovery sleep to the residual variation, after between-subject variation has been removed. Change in BTMs in response to insufficient sleep was assessed with the mixed model described above using BTM values measured every $2 \mathrm{~h}$ on two, 24-h profiles obtained at baseline and on the 6th night of sleep restriction. Change in BTMs in response to subsequent recovery sleep was assessed with the mixed model described above using eight BTM values starting with one value from each 24 -h serum collection through the end of the observational recovery phase. Circadian rhythmicity was included in the repeated measures model for all three BTMs as diurnal variation has been documented in prior studies [37-39]. Data from the repeated measures model are presented as estimate \pm standard error of the estimate (SEE) unless otherwise stated. Descriptive variables are presented as mean \pm standard deviation (SD) or number (percent), as appropriate. Paired $t$ tests were used to determine if changes in weight, physical activity, and sleep questionnaire scores were statistically significant. To assess the effect of heparin on BTM assessment, a paired $t$ test was performed on data for each BTM from the third time point of each 24-h collection profile when heparin was on at baseline but not during the sleep-restricted collection. A $p$ value $<0.05$ was considered statistically significant. All analyses were conducted using SAS software version 9.4. All figures were generated using GraphPad Prism 9.0 (GraphPad Software).

\section{Results}

Men were 21-40 years old at the time of inpatient admission (average $28.3 \pm 5.7$ years; Table 1 ). Race/ethnicity were determined by self-report as follows: Caucasian/Not Hispanic $(N=6)$, Asian/Not Hispanic $(N=4)$, Black/Not Hispanic $(N=2)$. On average, polysomnography-determined sleep duration was $404 \pm 42 \mathrm{~min}$ and $283 \pm 11 \mathrm{~min}$ on the baseline and sleep-restricted $24-\mathrm{h}$ profile nights, respectively. Consistent with imposed sleep restriction, sleepiness increased over the course of the inpatient stay $(\Delta \mathrm{ESS}=4.67 \pm 6.23, p=0.02)$. On average, participants had $7.26 \pm 0.7 \mathrm{~h}$ time in bed per $24 \mathrm{~h}$ during the 3 -week observational recovery phase of the protocol with an average time of sleep onset of 12:13 am and an average wake time of 7:41 am.

There were no statistical or clinically significant changes in PINP, osteocalcin, or $\beta$-CTX in response to six nights of insufficient sleep duration (Table 2; Fig. 2). Results were unchanged when data were re-analyzed after excluding the 
Table 1 Participant demographics

\begin{tabular}{ll}
\hline Age (years) & $28.3 \pm 5.7$ \\
\hline Race/Ethnicity & \\
Asian/Not Hispanic or Latino & $4(33.3 \%)$ \\
Black or African American/Not Hispanic or Latino & $2(16.7 \%)$ \\
White/Not Hispanic or Latino & $6(50 \%)$ \\
BMI (kg/m $\left.{ }^{2}\right)$ & $23.3 \pm 2.6$ \\
25-hydroxyvitamin D (ng/mL) & $29 \pm 12.1$ \\
Calcium (mg/dL) & $9.7 \pm 0.3$ \\
Creatinine (mg/dL) & $0.86 \pm 0.10$ \\
L-spine BMD (g/cm $\left.{ }^{2}\right)$ & $1.073 \pm 0.155$ \\
L-spine Z-score & $-0.2 \pm 1.5$ \\
Total Hip BMD (g/cm ${ }^{2}$ ) & $1.043 \pm 0.137$ \\
Total Hip Z-score & $0.0 \pm 0.9$ \\
Apnea-Hypopnea Index (AHI) & $7.7 \pm 4.2$ \\
PSQI Score (at screening) & $3.1 \pm 0.9$ \\
Epworth Sleepiness Scale (at baseline) & $4.3 \pm 3.0$ \\
\hline
\end{tabular}

Data are presented as mean \pm standard deviation (SD) or mean (\%) BMI body mass index, $L$-spine Lumbar spine, $B M D$ bone mineral density, PSQI Pittsburgh Sleep Quality Index

Table 2 Baseline concentrations and effect of sleep restriction intervention on bone turnover markers $(N=12)$

\begin{tabular}{lcc}
\hline & $\begin{array}{l}\text { Baseline bio- } \\
\text { marker concentra- } \\
\text { tion* } \\
\text { Estimate } \pm \text { SEE }\end{array}$ & Effect of intervention \\
& Estimate \pm SEE, $p$ value \\
\hline PINP $(\mu \mathrm{g} / \mathrm{L})$ & $72.1 \pm 4.4$ & $2.7 \pm 4.2,0.53$ \\
$\beta$-CTX $(\mathrm{ng} / \mathrm{L})$ & $197 \pm 50$ & $-57 \pm 30,0.10$ \\
Osteocalcin $(\mathrm{ng} / \mathrm{mL})$ & $20.8 \pm 2.0$ & $0.7 \pm 1.6,0.66$ \\
\hline
\end{tabular}

Data presented as estimate \pm standard error of the estimate (SEE)

*Baseline biomarker concentration calculated using all data points in first 24-h sample collection

participant who experienced the protocol deviation (data not shown).

Although there was no change in BTMs in response to sleep restriction, the trajectory of BTM change with resumption of normal, unprescribed sleep schedule was examined for 3 weeks after discharge to determine if the inpatient environment influenced BTM levels during both the baseline and post-intervention assessments. There were no significant changes in PINP, osteocalcin, or $\beta$-CTX from the inpatient stay through 3 weeks of recovery sleep (all $p \geq$ 0.63 ; Table 3). Results were unchanged when the analysis was repeated after excluding the first $24-\mathrm{h}$ baseline profile data point (data not shown).

On average, daily physical activity levels assessed by Fitbit and wrist actigraphy were slightly lower during the inpatient week compared to the outpatient run-in week (575 \pm 648 fewer steps $p=0.01$ and $11.4 \% \pm 10.0 \%$ lower activity $p<0.01$, respectively; Table 4$)$. However, there were no statistically significant differences in activity level between the two 24-h collection days by wrist actigraphy or Fitbit (Table 4). On average, weight was stable from admission to the end of sleep restriction $(\Delta$ weight $=-0.55 \pm 0.91 \mathrm{~kg}$, $p=0.06)$. There were no clinically or statistically significant differences between the two means at minute $240(\sim$ midnight in Fig. 2) when heparin was on during the baseline collection but off during the subsequent sleep-restricted collection in $\beta$-CTX $(41.9 \pm 146.8 \mathrm{ng} / \mathrm{L}, p=0.343)$, PINP $(1.3$ $\pm 24.3 \mathrm{~g} \mu / \mathrm{L}, p=0.858)$, or osteocalcin $(5.8 \pm 9.2 \mathrm{ng} / \mathrm{mL}$, $p=0.053$ ) levels.

\section{Discussion}

This was the first human study designed to evaluate BTM change after exposure to six nights of insufficient $5 \mathrm{~h} /$ night sleep duration, the mechanistic role of energy intake, physical activity, and posture, and the ability of BTMs to recovery with resumption of normal sleep/wake schedules. No statistically or clinically significant [40] changes in BTMs were identified in response to insufficient sleep when energy balance, physical activity, and posture were controlled, or during subsequent resumption of habitual sleep/wake schedules over 3 weeks.

The lack of BTM changes in response to insufficient sleep duration observed in this protocol could inform underlying mechanisms by which sleep disruption can alter bone metabolism, including the role of energy intake/weight change, physical activity, and posture during insufficient sleep. In the current study, caloric intake was increased 7\% during sleep restriction to account for increased energy expenditure observed in the previous sleep restriction studies [35, 36]. As a result, participant weight was stable from the beginning to the end of the protocol. This differs from prior studies that identified significant differences in BTMs in response to sleep restriction and circadian disruption in humans [22-24, 26] and animals [12] in which food intake was not increased and/or weight loss occurred with the sleep/circadian intervention. In the sleep restriction study by Xu et al. animals had access to food ad libitum, but sleep-restricted rats gained less weight than controls [13]. This theory is consistent with another recent study that did not observe BTM change in response to nine nights of insufficient sleep with or without recovery sleep in which food intake was ad libitum [41]. These data are consistent with the theory that weight loss (or less weight gain) during sleep restriction contributes mechanistically to BTM changes in response to sleep restriction. Furthermore, the current study had no significant changes in physical activity level or posture between the two 24-h collection days, by design. Prior studies observed an increase 
Fig. 2 Serum BTM 24-h profiles at baseline and during sleep restriction (SR). Data from 24-h serum BTM profiles obtained at baseline (blue) and on the last night of sleep restriction (SR; purple) are displayed for a PINP $(\mu \mathrm{g} / \mathrm{L})$, b osteocalcin $(\mathrm{ng} / \mathrm{mL})$, and $\mathbf{c} \beta$-CTX (ng/L). $X$-axis represents approximate relative clock time based on average bedtime and waketime of participants self-selected 8-h sleep opportunity. Upsidedown triangles along the $x$-axis represent meal times including breakfast ( 8 a.m.), lunch $(\sim 1$ p.m.), dinner ( 6 p.m.), and snack ( 8 p.m.). Each graph displays raw average values for each bone biomarker obtained every $2 \mathrm{~h}$ as open light blue circles (baseline) or open purple squares (SR) with one-way standard error of the estimate (SEE) bars. Fitted 24-h curves are depicted as a dotted light blue line for baseline and a solid purple line for SR. Time in bed sleep opportunities at baseline and during SR are represented by horizontal light blue and purple bars, respectively, at the top of each graph
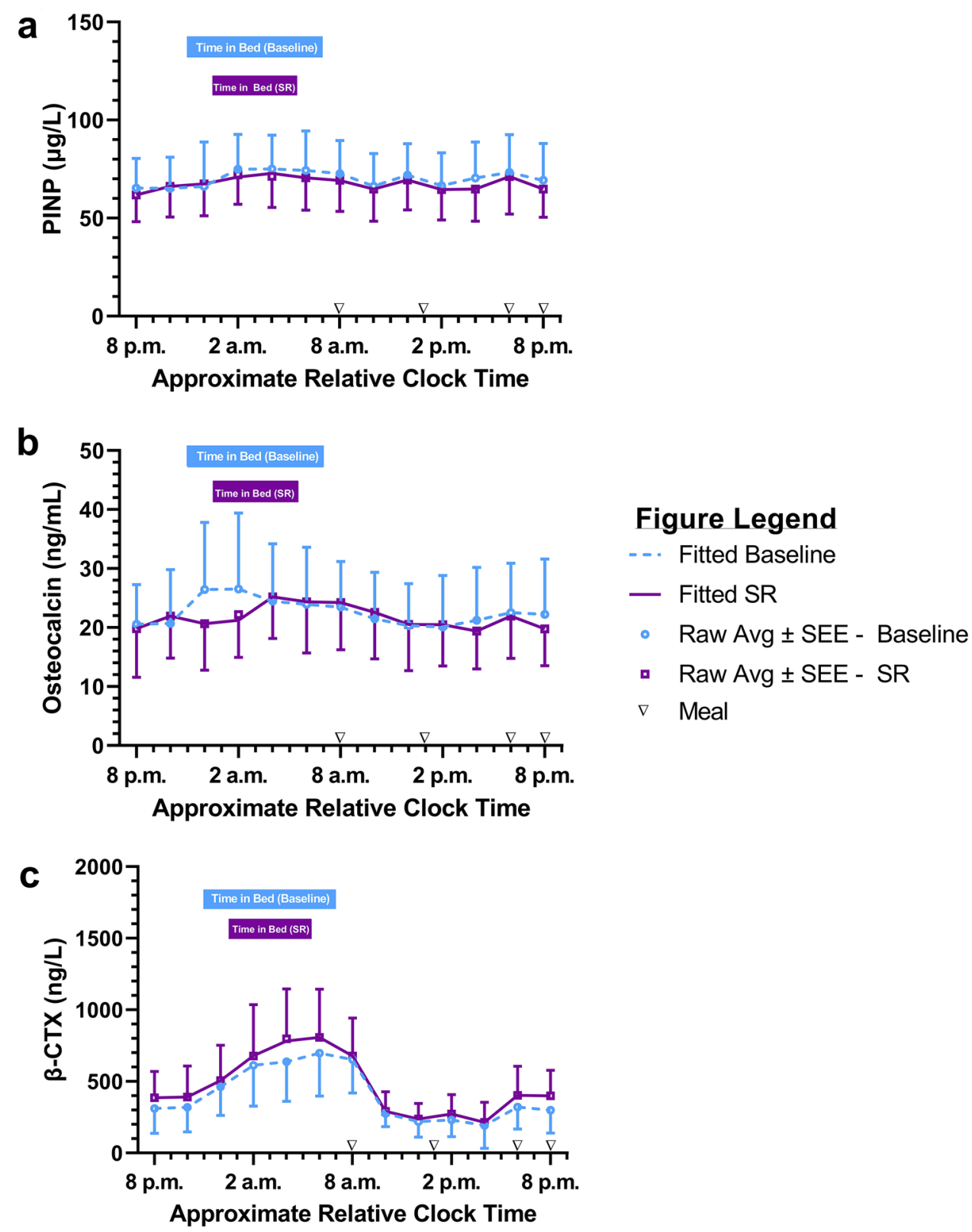

in physical activity from the beginning to the end of the inpatient protocol [23, 24], which may have contributed to the weight loss and BTM changes. It is possible that inherent differences in the inpatient environment in which these studies were conducted, compared to the habitual home environment, contribute to BTM changes independent of the sleep or circadian disruption imposed. However, the lack of BTM change observed from the end of the inpatient stay to the end of the 3-week observation phase in the current protocol argues against any effect of the inpatient environment itself on BTM levels in this study.

The sleep restriction imposed in the current study may not have been sufficient to provoke significant changes in bone metabolism. The magnitude of sleep restriction $(5 \mathrm{~h} /$ night) was similar to prior human studies in which significant BTM changes were observed within the first 10 days of exposure to sleep restriction ( $\sim 5.5 \mathrm{~h}$ per $24 \mathrm{~h}$ day) with concurrent circadian disruption [23-25]. Furthermore, a study by Staab et al. demonstrated significant changes in BTMs after just one night of $2 \mathrm{~h} /$ night sleep restriction [22]. These data suggest that the magnitude and duration of sleep restriction in the current protocol should have been sufficient to provoke significant BTM changes. However, the previous human studies imposed more significant circadian disruption (by using a forced desynchrony protocol and significantly shorter sleep) than the current study, potentially explaining the different results. In fact, in a recent study, statistically non-significant BTM changes in response to 
insufficient sleep were correlated with morning circadian misalignment [41]. The 24-h profiles of osteocalcin and $\beta$-CTX appeared to briefly diverge during the prescribed sleep opportunities (Fig. 2) and occurred in the same directions as observed in prior studies (i.e., osteocalcin decreased and $\beta$-CTX increased in the sleep-restricted profile). The clinical significance of these brief divergences between the two 24-h profiles and their possible relationship to longer duration of heparin exposure during baseline compared to the sleep-restricted day requires further research. Prior literature [42] and data from this study suggest that the very low-dose heparin used only during two sleep opportunities in this study did not significantly affect bone turnover marker assays or levels.

This study to investigate changes in bone metabolism in response to insufficient sleep had several strengths including a rigorous study design, controlled inpatient environment, matching of physical activity, and dietary control resulting in stable body weight. However, there were some limitations. First, a protocol deviation resulted in slightly longer sleep duration on one night in one participant. However, results were unchanged when data were re-analyzed without data from that participant. Second, only a relatively small cohort $(N=12)$ of young, healthy, physically active men was studied. However, prior data [23, 24] suggested the greatest magnitude of BTM change in response to sleep and circadian disruption occurred in younger individuals and a priori power calculations suggested the study was adequately powered to observe clinically significant changes in PINP in this racially diverse cohort. It remains possible that the study was underpowered or that the diversity of the cohort limited the ability to detect significant differences in certain subgroups. Although the skeletal response to sleep restriction has not been shown to differ by race/ethnicity $[18,25$, 41], this should be explored in future studies. Third, the similar physical activity levels and bedrest imposed during shortened sleep may not be commonly experienced when sleep restriction occurs in real life. However, controlling these factors may provide mechanistic insight into how sleep restriction alters bone metabolism. Fourth, the controlled diet in this study may not accurately replicate real-life changes in food intake in response to sleep restriction, as previous studies have demonstrated increased caloric intake with preference for carbohydrate foods during sleep restriction $[35,36]$. Although calorie intake was increased during sleep restriction in the current study, participants did not have ad libitum access to food. However, as discussed above, this may indicate that changes in food intake and subsequent weight change play a mechanistic role in the detrimental BTM changes observed in previous animal/human studies [12, 13, 22-24, 26]. 
Table 4 Change in physical activity as measured by fitbit charge 3 and actiwatch 2 from (a) outpatient week to inpatient week, and (b) baseline 24-h (h) serum collection to sleeprestricted 24-h serum collection
Steps per day

(fitbit charge 3)

(a) Daily average \pm standard deviation (SD)

$\begin{array}{lll}\text { Inpatient stay } & 13,731 \pm 3161 & 266,043 \pm 78,342 \\ \text { Outpatient run-in week } & 14,306 \pm 3052 & 320,012 \pm 80,179 \\ \text { Difference }(p \text {-value }) & -575 \pm 648(p=0.01) & -34,682 \pm 32,513(p=0.005) \\ \text { Percent change }(p \text { value }) & -4.2 \% \pm 4.4 \%(p=0.01) & -11.4 \% \pm 10.0 \%(p=0.005) \\ \text { (b) Average } \pm \text { SD during } 24-h \text { serum profile collection } & \\ \text { Sleep Restricted } & 12,185 \pm 3360 & 254,950 \pm 65,007 \\ \text { Baseline } & 12,763 \pm 2711 & 277,636 \pm 82,655 \\ \text { Difference }(p \text { value }) & -578 \pm 3057(p=0.53) & -18,343 \pm 74,818(p=0.44) \\ \text { Percent change }(p \text { value }) & -3.3 \% \pm 19.5 \%(p=0.53) & -3.5 \% \pm 24.5 \%(p=0.44)\end{array}$

In conclusion, no significant changes were observed in serum markers of bone formation or resorption after six nights of insufficient sleep duration of $5 \mathrm{~h} /$ night or subsequent recovery sleep in young healthy men. It is possible that changes in body weight and/or physical activity, which did not occur by design in the current study, may have been mechanistically linked to the changes in BTMs observed in response to insufficient sleep duration in previous studies.

Acknowledgements This research was supported by R03 AR074509 (CMS), K23 AR070275 (CMS), and the NIH/NCATS Colorado CTSA Grant number UL1TR002535. Contents are the authors' sole responsibility and do not necessarily represent official NIH views. CAR is supported by K01 DK113063. KPW received support from NIH Grants R01HL135598, R01HL131458, R01HD087707, R01DK114272, R01DK115502, U01HL150596, a Pac-12 Grant Application, and ONR MURI N00014-15-1-2809 during the time of this research. We thank Rebecca Baldermann for her expertise and input as the University of Colorado CTRC Lab Manager.

Author Contributions CMS, KPW, and WMK: study concept and design; CMS and EJT: data collection and study performance; PS, EJT, and CAR: data analysis; CMS: funding acquisition for this analysis; CMS, KPW, and WMK: data interpretation; CMS: drafting manuscript; CMS, PS, EJT, CAR, KPW, and WMK: manuscript revisions and approval of final manuscript; PS, EJT, and CAR: responsibility for integrity of data analysis.

Funding This research was supported by R03 AR074509 (CMS), K23 AR070275 (CMS), and the NIH/NCATS Colorado CTSA Grant number UL1TR002535. Contents are the authors' sole responsibility and do not necessarily represent official NIH views. CAR is supported by K01 DK113063. KPW received support from NIH Grants R01HL135598, R01HL131458, R01HD087707, R01DK114272, R01DK115502, U01HL150596, a Pac-12 Grant Application, and ONR MURI N0001415-1-2809 during the time of this research.

Data Availability Data from this study are posted on ClinicalTrials.gov. Other study data are not publicly available but are available from the corresponding author on reasonable request.
Code Availability The custom SAS code used to generate these results are not publicly available but are available from the corresponding author on reasonable request.

\section{Declarations}

Conflict of interest CMS, PS, EJT, CAR, and WMK have nothing to disclose. KPW reports research support from the NIH, Office of Naval Research, Pac-12. Financial relationships: consulting fees Circadian Therapeutics, LTD., Circadian Biotherapies, Inc., Philips Respironics. Board of Directors: Sleep Research Society.

Ethical Approval All procedures performed in studies involving human participants were in accordance with the ethical standards of the institutional and/or national research committee and with the 1964 Helsinki declaration and its later amendments or comparable ethical standards.

Informed Consent Informed consent was obtained from all individual participants included in the study.

\section{References}

1. Eckel RH, Depner CM, Perreault L, Markwald RR, Smith MR, McHill AW, Higgins J, Melanson EL, Wright KP Jr (2015) Morning circadian misalignment during short sleep duration impacts insulin sensitivity. Curr Biol 25(22):3004-3010. https://doi.org/ 10.1016/j.cub.2015.10.011

2. Buxton OM, Pavlova M, Reid EW, Wang W, Simonson DC, Adler GK (2010) Sleep restriction for 1 week reduces insulin sensitivity in healthy men. Diabetes 59(9):2126-2133. https://doi.org/10. 2337/db09-0699

3. Buxton OM, Marcelli E (2010) Short and long sleep are positively associated with obesity, diabetes, hypertension, and cardiovascular disease among adults in the United States. Soc Sci Med 71(5):1027-1036. https://doi.org/10.1016/j.socscimed.2010.05. 041

4. Cappuccio FP, D’Elia L, Strazzullo P, Miller MA (2010) Quantity and quality of sleep and incidence of type 2 diabetes: a systematic review and meta-analysis. Diabetes Care 33(2):414-420. https:// doi.org/10.2337/dc09-1124

5. Arble DM, Bass J, Behn CD, Butler MP, Challet E, Czeisler C, Depner CM, Elmquist J, Franken P, Grandner MA, Hanlon EC, 
Keene AC, Joyner MJ, Karatsoreos I, Kern PA, Klein S, Morris CJ, Pack AI, Panda S, Ptacek LJ, Punjabi NM, Sassone-Corsi P, Scheer FA, Saxena R, Seaquest ER, Thimgan MS, Van Cauter E, Wright KP (2015) Impact of sleep and circadian disruption on energy balance and diabetes: a summary of workshop discussions. Sleep 38(12):1849-1860. https://doi.org/10.5665/sleep. 5226

6. Kecklund G, Axelsson J (2016) Health consequences of shift work and insufficient sleep. BMJ 355:i5210. https://doi.org/10.1136/ bmj.i5210

7. Wu Y, Zhai L, Zhang D (2014) Sleep duration and obesity among adults: a meta-analysis of prospective studies. Sleep Med 15(12):1456-1462. https://doi.org/10.1016/j.sleep.2014.07.018

8. Cappuccio FP, Cooper D, D’Elia L, Strazzullo P, Miller MA (2011) Sleep duration predicts cardiovascular outcomes: a systematic review and meta-analysis of prospective studies. Eur Heart J 32(12):1484-1492. https://doi.org/10.1093/eurheartj/ehr007

9. Yang X, Chen H, Li S, Pan L, Jia C (2015) Association of Sleep Duration with the Morbidity and Mortality of Coronary Artery Disease: A Meta-analysis of Prospective Studies. Heart Lung Circ 24(12):1180-1190. https://doi.org/10.1016/j.hlc.2015.08.005

10. Cappuccio FP, D’Elia L, Strazzullo P, Miller MA (2010) Sleep duration and all-cause mortality: a systematic review and metaanalysis of prospective studies. Sleep 33(5):585-592. https://doi. org/10.1093/sleep/33.5.585

11. Gallicchio L, Kalesan B (2009) Sleep duration and mortality: a systematic review and meta-analysis. J Sleep Res 18(2):148-158. https://doi.org/10.1111/j.1365-2869.2008.00732.x

12. Everson CA, Folley AE, Toth JM (2012) Chronically inadequate sleep results in abnormal bone formation and abnormal bone marrow in rats. Exp Biol Med 237(9):1101-1109. https://doi.org/10. 1258/ebm.2012.012043

13. Xu X, Wang L, Chen L, Su T, Zhang Y, Wang T, Ma W, Yang F, Zhai W, Xie Y, Li D, Chen Q, Fu X, Ma Y, Zhang Y (2016) Effects of chronic sleep deprivation on bone mass and bone metabolism in rats. J Orthop Surg Res 11(1):87. https://doi.org/ 10.1186/s13018-016-0418-6

14. Ochs-Balcom HM, Hovey KM, Andrews C, Cauley JA, Hale L, Li W, Bea JW, Sarto GE, Stefanick ML, Stone KL, Watts NB, Zaslavsky O, Wactawski-Wende J (2020) Short sleep is associated with low bone mineral density and osteoporosis in the women's health initiative. J Bone Min Res 35(2):261-268. https://doi.org/ 10.1002/jbmr.3879

15. Swanson CM (2021) Sleep disorders and osteoporosis. In: Dempster DW, Cauley JA, Bouxsein ML, Cosman F (eds) Marcus and Feldman's osteoporosis, 5th edn. Academic Press, Cambridge, pp 1459-1471

16. Swanson CM, Kohrt WM, Buxton OM, Everson CA, Wright KP Jr, Orwoll ES, Shea SA (2018) The importance of the circadian system \& sleep for bone health. Metabolism 84:28-43. https://doi. org/10.1016/j.metabol.2017.12.002

17. Wang D, Ruan W, Peng Y, Li W (2018) Sleep duration and the risk of osteoporosis among middle-aged and elderly adults: a dose-response meta-analysis. Osteoporos Int 29(8):1689-1695. https://doi.org/10.1007/s00198-018-4487-8

18. Cauley JA, Hovey KM, Stone KL, Andrews CA, Barbour KE, Hale L, Jackson RD, Johnson KC, LeBlanc ES, Li W, Zaslavsky O, Ochs-Balcom H, Wactawski-Wende J, Crandall CJ (2019) Characteristics of self-reported sleep and the risk of falls and fractures: the women's health initiative (WHI). J Bone Min Res 34(3):464-474. https://doi.org/10.1002/jbmr.3619

19. Swanson CM, Blatchford PJ, Orwoll ES, Cauley JA, LeBlanc ES, Fink HA, Wright KP Jr, Wierman ME, Kohrt WM, Stone KL, Study of Osteoporotic F (2019) Association between objective sleep duration and bone mineral density in older postmenopausal women from the study of osteoporotic fractures (SOF).
Osteoporos Int 30(10):2087-2098. https://doi.org/10.1007/ s00198-019-05007-5

20. Centers for Disease C (2014) insufficient sleep is a public health epidemic. http://www.cdc.gov/features/dssleep/index.html\#Refer ences

21. Matthews KA, Croft JB, Liu Y, Lu H, Kanny D, Wheaton AG, Cunningham TJ, Khan LK, Caraballo RS, Holt JB, Eke PI, Giles WH, FtCfDCa P (2017) Health-related behaviors by urban-rural county classification-United States, 2013. MMWR Surveill Summ 66(5): 1

22. Staab JS, Smith TJ, Wilson M, Montain SJ, Gaffney-Stomberg E (2019) Bone turnover is altered during $72 \mathrm{~h}$ of sleep restriction: a controlled laboratory study. Endocrine 65(1):192-199. https:// doi.org/10.1007/s12020-019-01937-6

23. Swanson C, Shea SA, Wolfe P, Cain SW, Munch M, Vujovic N, Czeisler CA, Buxton OM, Orwoll ES (2017) Bone turnover markers after sleep restriction and circadian disruption: a mechanism for sleep-related bone loss in humans. J Clin Endocrinol Metab 102:3722-3730. https://doi.org/10.1210/jc.2017-01147

24. Swanson CM, Shea SA, Kohrt WM, Wright KP, Cain SW, Munch M, Vujovic N, Czeisler CA, Orwoll ES, Buxton OM (2020) Sleep restriction with circadian disruption negatively alter bone turnover markers in women. J Clin Endocrinol Metab. https://doi.org/10. 1210/clinem/dgaa232

25. Swanson CM, Kohrt WM, Wolfe P, Wright KP Jr, Shea SA, Cain SW, Munch M, Vujovic N, Czeisler CA, Orwoll ES, Buxton OM (2019) Rapid suppression of bone formation marker in response to sleep restriction and circadian disruption in men. Osteoporos Int 30(12):2485-2493. https://doi.org/10.1007/s00198-019-05135-y

26. Hughes JM, Smith MA, Henning PC, Scofield DE, Spiering BA, Staab JS, Hydren JR, Nindl BC, Matheny RW Jr (2014) Bone formation is suppressed with multi-stressor military training. Eur J Appl Physiol. https://doi.org/10.1007/s00421-014-2950-6

27. Limited IM (2016) What is WatchPAT? http://www.itamar-medic al.com/patients-watchpat/.

28. ActiWatch 2 activity monitor. http://www.usa.philips.com/ healthcare/product/HC1044809/actiwatch-2-activity-monitor. Accessed 3 Aug 2017

29. Berry RB, L. AC, M. HS, Lloyd RM, Plante DT, Quan SF, Troester MM, Baughn BV, Medicine ftAAoS (2018) The AASM manual for the scoring of sleep and associated events: rules, terminology and technical specifications, version 2.5. Darien, IL. https://aasm.org/resources/pdf/scoring-manual-preface.pdf

30. Buysse DJ, Reynolds CF 3rd, Monk TH, Berman SR, Kupfer DJ (1989) The Pittsburgh Sleep Quality Index: a new instrument for psychiatric practice and research. Psychiatry Res 28(2):193-213

31. Johns MW (1991) A new method for measuring daytime sleepiness: the Epworth sleepiness scale. Sleep 14(6):540-545

32. Johns MW (2019) The Epworth Sleepiness Scale. http://epwor thsleepinessscale.com

33. Patel SR, Weng J, Rueschman M, Dudley KA, Loredo JS, Mossavar-Rahmani Y, Ramirez M, Ramos AR, Reid K, Seiger AN, Sotres-Alvarez D, Zee PC, Wang R (2015) Reproducibility of a standardized actigraphy scoring algorithm for sleep in a US hispanic/latino population. Sleep 38(9):1497-1503. https://doi.org/ 10.5665/sleep.4998

34. Mifflin MD, St Jeor ST, Hill LA, Scott BJ, Daugherty SA, Koh YO (1990) A new predictive equation for resting energy expenditure in healthy individuals. Am J Clin Nutr 51(2):241-247. https://doi. org/10.1093/ajcn/51.2.241

35. Jung CM, Melanson EL, Frydendall EJ, Perreault L, Eckel RH, Wright KP (2011) Energy expenditure during sleep, sleep deprivation and sleep following sleep deprivation in adult humans. J Physiol 589(Pt 1):235-244. https://doi.org/10.1113/jphysiol.2010. 197517 
36. Markwald RR, Melanson EL, Smith MR, Higgins J, Perreault L, Eckel RH, Wright KP Jr (2013) Impact of insufficient sleep on total daily energy expenditure, food intake, and weight gain. Proc Natl Acad Sci USA 110(14):5695-5700. https://doi.org/10.1073/ pnas. 1216951110

37. Swanson C, Shea SA, Wolfe P, Markwardt S, Cain SW, Munch M, Czeisler CA, Orwoll ES, Buxton OM (2017) 24-hour profile of serum sclerostin and its association with bone biomarkers in men. Osteoporos Int 28:3205-3213. https://doi.org/10.1007/ s00198-017-4162-5

38. Qvist P, Christgau S, Pedersen BJ, Schlemmer A, Christiansen C (2002) Circadian variation in the serum concentration of C-terminal telopeptide of type I collagen (serum CTx): effects of gender, age, menopausal status, posture, daylight, serum cortisol, and fasting. Bone 31(1):57-61

39. Redmond J, Fulford AJ, Jarjou L, Zhou B, Prentice A, Schoenmakers I (2016) Diurnal rhythms of bone turnover markers in three ethnic groups. J Clin Endocrinol Metab 101(8):3222-3230. https://doi.org/10.1210/jc.2016-1183
40. Eastell R, Krege JH, Chen P, Glass EV, Reginster JY (2006) Development of an algorithm for using PINP to monitor treatment of patients with teriparatide. Curr Med Res Opin 22(1):61-66. https://doi.org/10.1185/030079905X75096

41. Depner CM, Rice JD, Tussey EJ, Eckel RH, Bergman BC, Higgins JA, Melanson EL, Kohrt WM, Wright KP Jr, Swanson CM (2021) Bone turnover marker responses to sleep restriction and weekend recovery sleep. Bone 152:116096. https://doi.org/10.1016/j.bone. 2021.116096

42. van der Wiel HE, Lips P, Huijgens PC, Netelenbos JC (1993) Effects of short-term low-dose heparin administration on biochemical parameters of bone turnover. Bone Miner 22(1):27-32 . https://doi.org/10.1016/s0169-6009(08)80078-5

Publisher's Note Springer Nature remains neutral with regard to jurisdictional claims in published maps and institutional affiliations. 